[Technical Paper]

\title{
Variations of Temperature Sensitivity Measurements of Several Packages Under Different Environments
}

\author{
Tomoaki Hara, Wasanthamala Badalawa, and Yafei Luo \\ Mentor Graphics Japan, Trust Tower 20F, Kita-Shinagawa 4-7-35, Shinagawa-ku, Tokyo 140-0001, Japan
}

(Received July 31, 2015; accepted December 7, 2015)

\begin{abstract}
Temperature sensitivity is used to measure the junction temperature of semiconductor devices without using external temperature sensors. Temperature sensitivity may vary due to the measurement environments, which affects the accuracy of the junction temperature. A single sided cold plate is widely used for thermal transient measurement. It would be useful if a single sided cold plate can be used not only for thermal transient measurements but also for temperature sensitivity measurements. However, it is presumed that temperature sensitivity measurement is not reliable when a single sided cold plate is used. It is due to the temperature gradient between the cold plate and the ambient, through the junction of the semiconductor. Unless the device is put inside the temperature controlled chamber, it is hard to eliminate the temperature gradient. However, this fact hasn't been well recognized. In order to clarify whether single sided cold plate has a chance for the temperature sensitivity measurement or not, this paper presents the results of several temperature sensitivity measurements using 2 types of temperature controlled environments: a single sided cold plate and a temperature controlled chamber. Also, we have tested with several packages such as TO-220, TO-264, SOT-227, and D3 package, to see how it varies. We have come to the conclusion that it is better to use a temperature controlled chamber to measure temperature sensitivity, while some devices allow us to use a single sided cold plate as well. The differences due to the devices and environments are discussed by using Computational Fluid Dynamics (CFD) simulation, visualizing the temperature gradient inside the packages.
\end{abstract}

Keywords: Thermal Sensitivity, Thermal Analysis, CFD Simulation, Thermal Transient Test, Structure Function

\section{Introduction}

Thermal transient test[1] is a widely-used standard measurement method for non-destructive investigation of heat flow path from the heat source to the ambient of semiconductor devices. The thermal transient test is performed not only to obtain the heat flow path as an RC - model of thermal systems, but also to obtain Junction-to-Case thermal resistance $\left(R_{t h-j c}\right)$,[2] to calibrate a thermal model of CFD simulators to compute more reliable simulation results, [3] to visualize the degradation process during a power cycling test of power devices, $[4,5]$ etc.

The temperature change of the junction temperature $\left(T_{j}\right)$ due to a known power step applied to the device is measured during the thermal transient test. It is common to use temperature sensitive parameters (TSP) to measure the $T_{j}$ without using external temperature sensors. [6] The temperature change $\Delta T_{j}$ can be described as follows:

$$
\Delta T_{j}=K \times \Delta T S P
$$

where $\triangle T S P(\mathrm{mV})$ is the change in temperature-sensitive parameter value, and $K(\mathrm{~K} / \mathrm{mV})$ is the constant defining relationship between changes in $T_{j}$ and TSP. The meaning of this TSP can be found in.[6-9] The accuracy of the temperature measurement relies on the accuracy of the TSP. A calibration of TSP is performed by measuring the voltage while applying a constant current to the device under test (DUT), and logging the value under several specified temperature values (Fig. 1). The temperature is controlled by either a temperature controlled chamber or a chiller connected to a cold plate. The temperature of those equipment are the reference for the TSP calibration.

Ideal way to calibrate TSP is to use a temperature controlled chamber setup (Fig. 2) instead of single sided cold plate setup (Fig. 3).

This is because temperature controlled chamber can 
control the temperature from all sides of the device. All sides of the device in the temperature controlled chamber are facing controlled temperature evenly so that there will be no temperature gradient between the junction and the controlled temperature when it reaches the steady state. On the other hand, the single sided cold plate can control the temperature only from one side of the device. Other sides of the device face the ambient temperature. This will cause a temperature gradient even if the device is placed in the environment for a long time and reached the steady state. Since we are relying on the temperature which is set to the chamber as the reference temperature, we must eliminate the temperature differences between the two

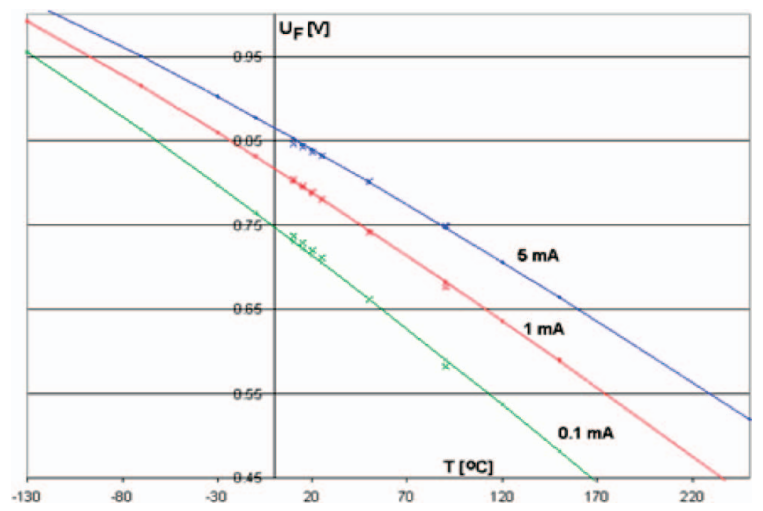

Fig. 1 Example of calibration results (Horizontal axis: junction temperature, Vertical axis: Voltage). during the TSP calibration to achieve more accurate results. Needless to say, the accuracy is relying on the accuracy of the chamber or the chiller attached to the single sided cold plate, too. If so, the more accurate the TSP is, the better results of the $T_{j}$ we get. There is no reason to use single sided cold plates for TSP calibration. However, especially for the larger devices to be calibrated, temperature controlled chamber might need much longer time to settle down to the desired temperature which ends up requiring a few hours, or more depending on the device to calibrate and how many temperature points to measure, for the calibration. Single sided cold plates can access to the device directly through the thermal interface material (TIM), it enables to lower the thermal resistance $R_{t h}$ $(\mathrm{K} / \mathrm{W})$ between the cold plates and the junction. This leads to the lower time constant $\tau$ (sec), which can be described as follows:

$$
\tau=R_{t h} \times C_{t h}
$$

$C_{t h}(\mathrm{Ws} / \mathrm{K})$ is thermal capacitance. The lower $\tau$ between the junction and the temperature controlled surface, the faster the stable state can be achieved. This is a benefit using single sided cold plates.

In general, thermal transient tests are performed by setting the device on a cold plate to draw the heat dissipated from the device during the test. Both TSP calibration and

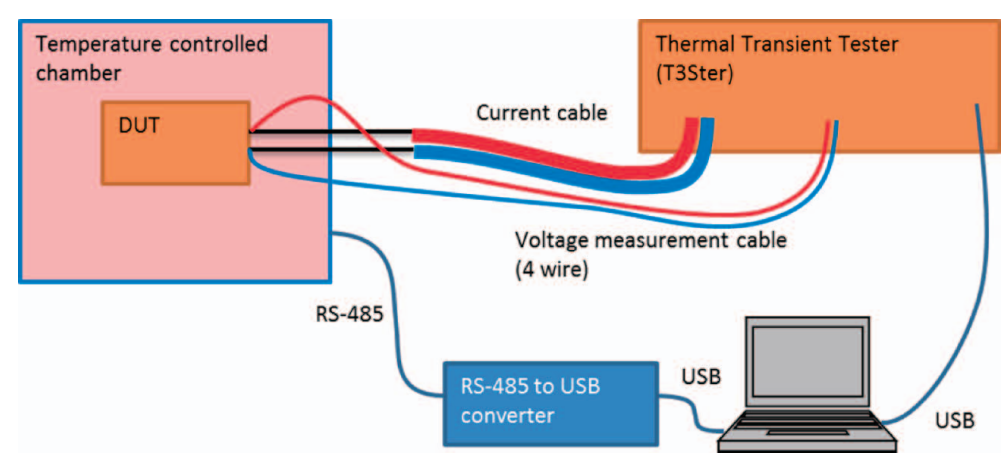

Fig. 2 Temperature controlled chamber setup.

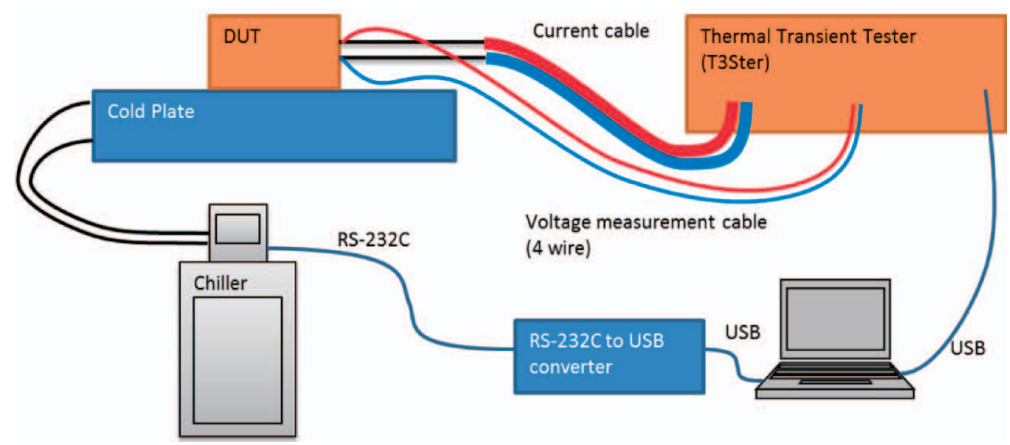

Fig. 3 Single sided cold plate setup. 
thermal transient test using the same jig would be another benefit using a single sided cold plate.

In this paper, we present the results of several temperature sensitivity measurements using 2 types of temperature controlled environments: a single sided cold plate and a temperature controlled chamber. Also, we have tested with several packages such as TO-220, TO-264, SOT-227, and D3 package, to see how it varies.

\section{Experiment Method}

\section{TSP calibration, considering the time constant from junction}

to temperature controlled chamber/single sided cold plate

Since the differences caused by TSP calibrating methods is the topic to be discussed in this paper, we needed to eliminate the differences in the measurement results due to how long it requires getting the device to the steady state from one temperature to another. If it is not long enough, junction of the device won't reach the target temperature and end up getting very poor results. If it is too long, it is not productive and waste of energy. It is not good for the environment.

\section{A method to judge $T_{j}$ reaches the steady state}

In general, the temperature drift $\Delta T_{\text {drift }}$ during time window $t_{T W}$ is used to decide whether $T_{j}$ has reached the steady state or not (Fig. 4). $\Delta T_{\text {drit }}$ is used because $\Delta T_{\text {err }}$ can't be yield directly before the calibration is done. If $\Delta T_{\text {drift }}$ is small enough to meet the level of accuracy to achieve, we decide $T_{j}$ has reached the steady state, or close enough to the target temperature.

Under this method, the temperature difference of the $T_{j}$ from the target temperature $\Delta T_{\text {err }}$ is expected to be smaller than $\Delta T_{\text {drift }}$. We can write the relationship as below:

$$
\Delta T_{\text {err }}(t) \leq \Delta T_{\text {drift }}(t)
$$

$\Delta T_{\text {drit }}$ can be calculated as below from Fig. 4:

$$
\Delta T_{\text {drift }}=\Delta T_{\text {err }}\left(t-t_{T W}\right)-\Delta T_{\text {err }}(t)
$$

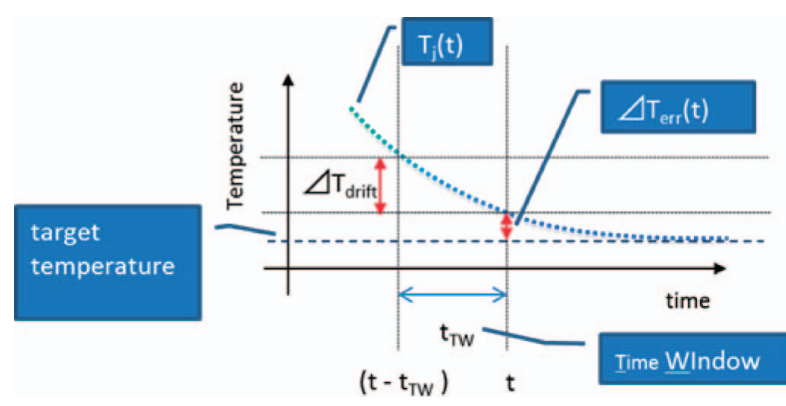

Fig. 4 TSP step response.
So the condition below should be met:

$$
2 \Delta T_{\text {drift }} \leq \Delta T_{\text {err }}\left(t-t_{T W}\right)
$$

$\Delta T_{e r r}(t)$ is proportional to $e^{-t / \tau}$ so the following equation can be yielded from Eq. (5):

$$
2 e^{-t / \tau} \leq e^{-\left(\left(t-t_{T W}\right) / \tau\right)}
$$

Then the condition $t_{T W}$ shown below should meet in order to achieve Eq. (3):

$$
t_{T W} \geq \ln (2) \times \tau
$$

\section{Obtaining $\tau$ from preliminary measurement}

Time constant $\tau$ for TSP calibration includes not only the specification of the device to be measured, but also the chamber/single sided cold plate. The $\tau$ of the device itself might be relatively easy to yield. However, TSP calibration requires the entire calibration system to be settled to the target temperature. The time constant $\tau$ which includes the entire system would be difficult to calculate.

In order to obtain $\tau$ which includes the entire system, we've performed a preliminary measurement for each device to decide $\tau$, to calculate desired $t_{T W}$. The preliminary measurement is done in the following order:

- Set the target device in the chamber/on the single sided cold plate and apply sensing current to the device. Use four-terminal sensing to measure the TSP or the voltage.

- Start the TSP measurement as soon as changing the target temperature of the chamber/single sided cold plate, $\Delta T=10(\mathrm{~K})$ or more, to see its step response, shown as Fig. 5. Horizontal and Vertical axes of Fig. 5 are logarithmic time (sec) and temperature change $(\mathrm{K})$ respectively.

- Calculate $\tau$ from the measured TSP step response, shown as Fig. 6.

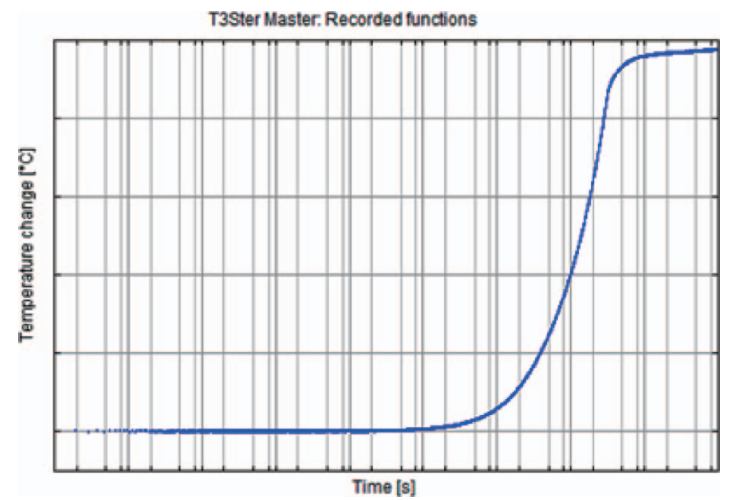

Fig. 5 Time constant $\tau$ calculated from TSP step response. 
TSP measurement and $\tau$ calculation were performed by T3Ster $^{\circledR}$.

For this example, calculated largest $\tau$ was 345 (sec). From Eq. (7), desired $t_{T W}$ should meet the condition below:

$$
t_{T W} \geq 239(\mathrm{sec})
$$

We used $t_{T W}=300(\mathrm{sec})$ for this device in this setup.

\section{Experiment}

TSP calibration was performed in the environment as shown in the Figs. 7 and 8. They show the environment of temperature controlled chamber and single sided cold plate respectively. The temperature of the single sided cold plate was controlled by a Refrigerated and Heating Circulator (Julabo F25-MA) which temperature stability is \pm 0.02 K. Five different devices were calibrated in this experiment (TO-220 full molded, TO-220, TO-264, SOT-227, and D3 package) and each result is shown in Figs. 9 to 13 accordingly. For the D3 package, it was too big to put into a small chamber (T3Ster Thermostat) which was used for other DUTs, a larger chamber (ESPEC SH-241) was used. The accuracy of a small chamber is $\pm 0.3 \mathrm{~K}$. For a larger

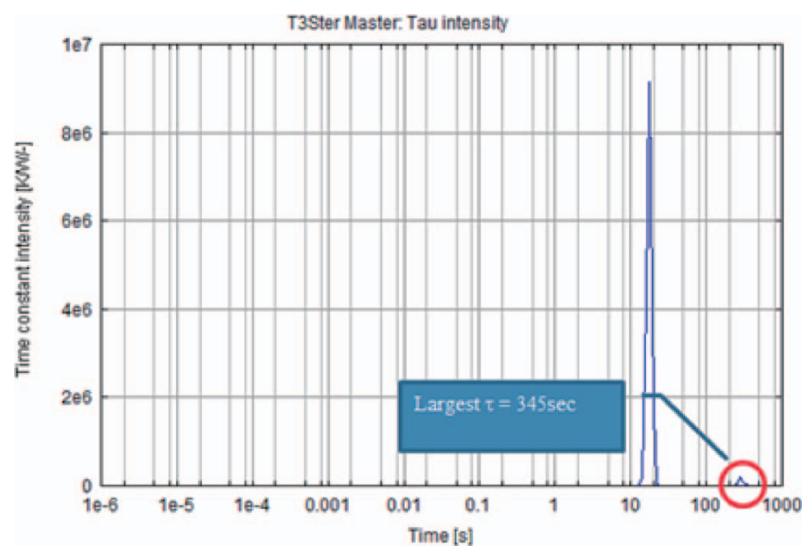

Fig. 6 Measurement environment of TSP calibration using a small chamber (T3Ster Thermostat).

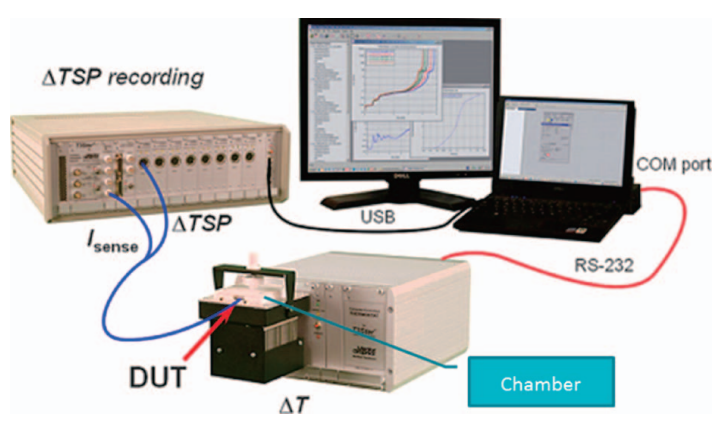

Fig. 7 Measurement environment of TSP calibration using single sided cold plate. chamber, temperature stability and accuracy is $\pm 0.5 \mathrm{~K}$ and $4 \mathrm{~K}$ respectively. TSP measurement was performed by a thermal transient tester (T3Ster) which resolution is \pm 0.01 K.

The differences of the calibrated results between temperature controlled chamber vs single sided cold plate is shown in Fig. 14. $R_{t h-j c}$ is also plotted as a reference.

In order to investigate the differences of two environments, CFD simulation was performed to see the temperature gradient which is generated when single sided cold

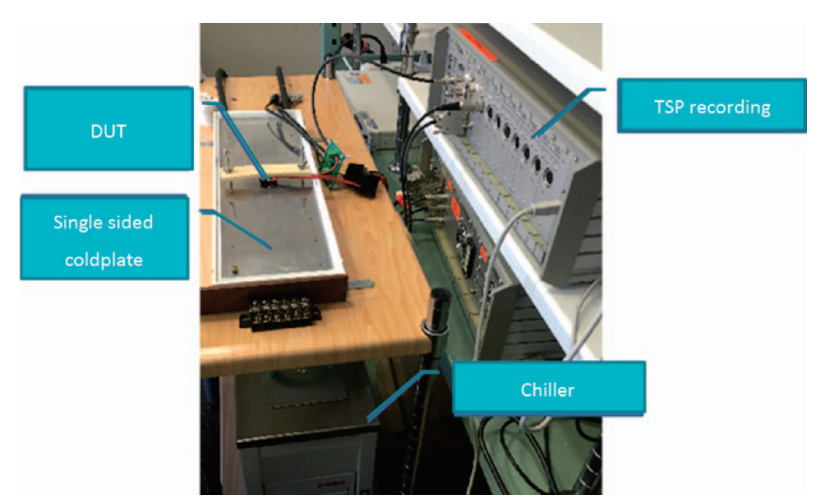

Fig. 8 TSP calibration results of TO-220 Full Molded device.

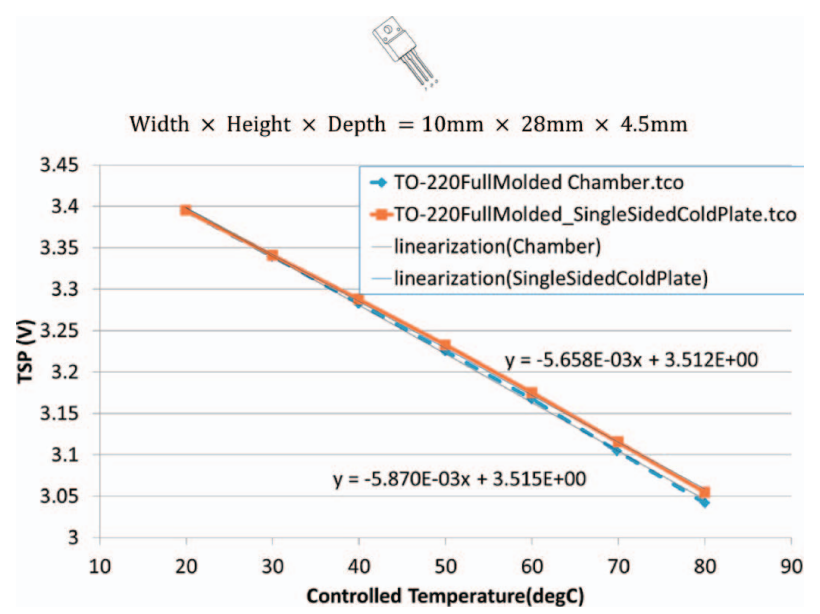

Fig. 9 TSP calibration results of TO-220 device.

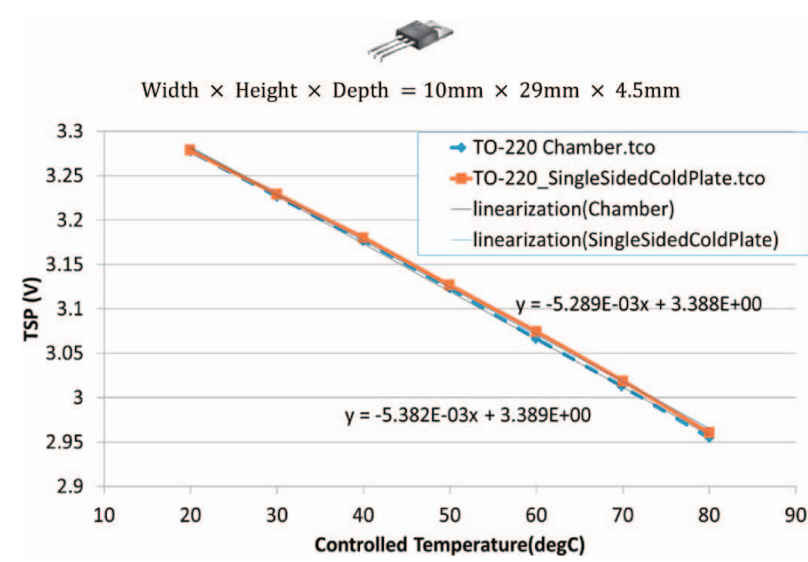

Fig. 10 TSP calibration results of TO-264 device. 


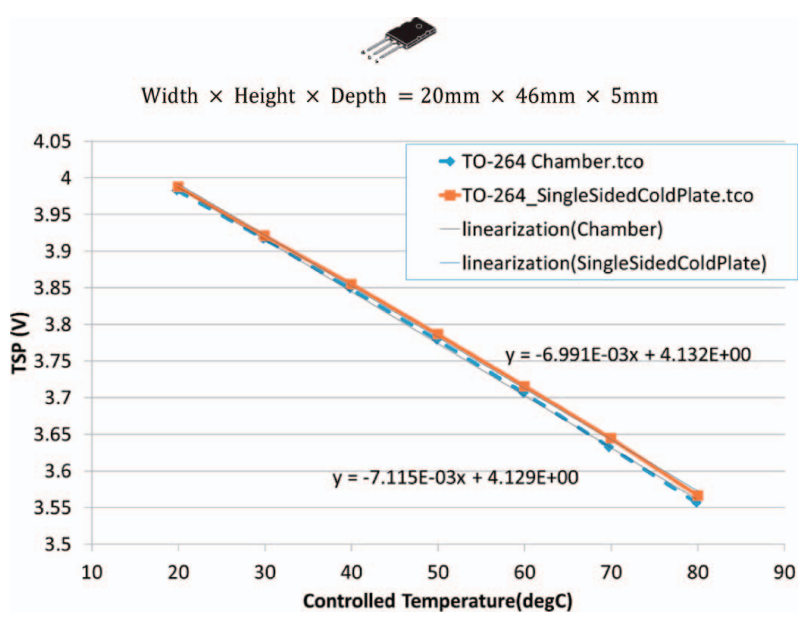

Fig. 11 TSP calibration results of SOT-227 device.

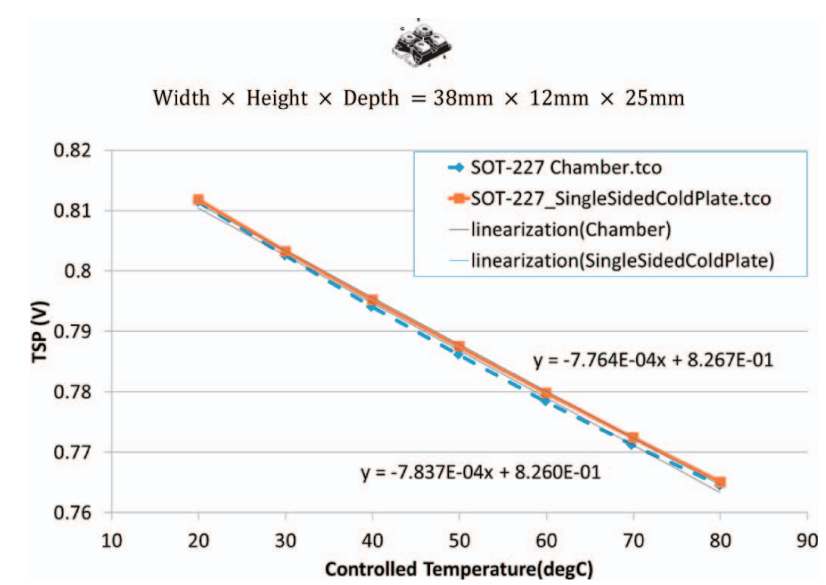

Fig. 12 TSP calibration results of D3 device.

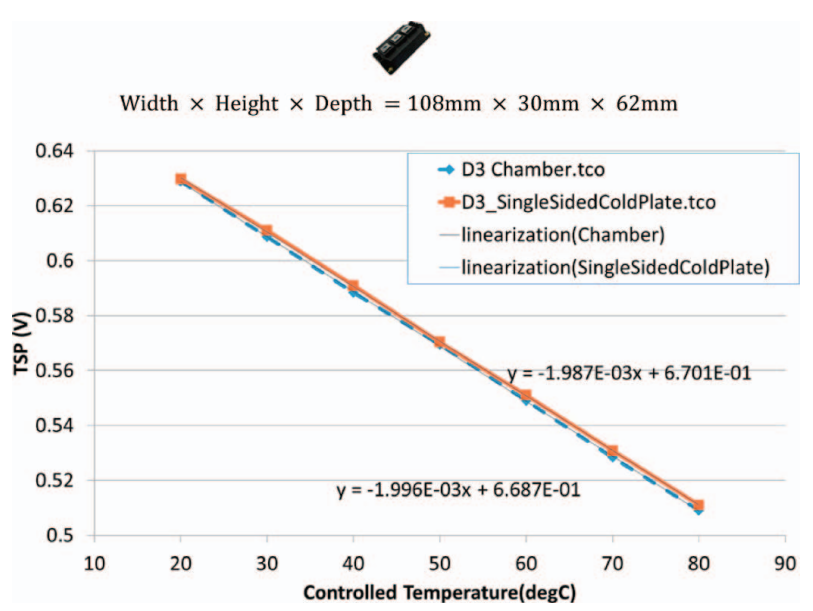

Fig. 13 TSP differences between temperature controlled chamber vs single sided cold plate (Left Axis) and $R_{t h-j c}$ written on the datasheets (Right Axis).

plate is used. The simulation result is shown in Figs. 15 and 16. For this experiment, FloTHERM 10.1 was used (Model size: $100 \mathrm{~mm}^{3}$, \# of grid cells: 468,779, Type of Solution: Flow And Heat Transfer, Turbulent, Pressure: 1atm, Default Radiant/Ambient Temperature: $25 \mathrm{degC}$, Transient Solution $(T=1,000 \mathrm{~s})$ ).

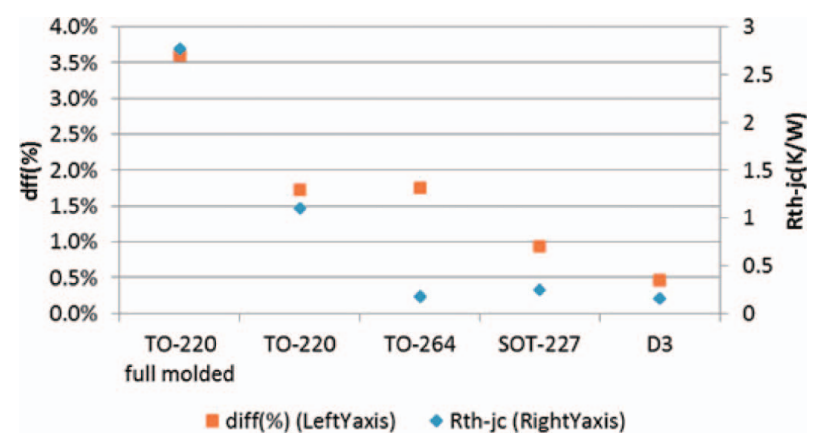

Fig. 14 Temperature gradient of TO-220 full molded package, on a single sided cold plate.

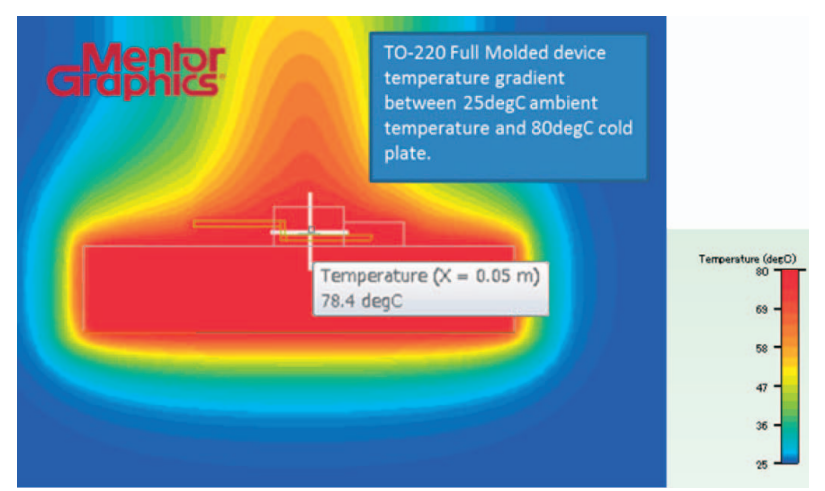

Fig. 15 Temperature gradient of TO-220 full molded package, in a temperature controlled chamber.

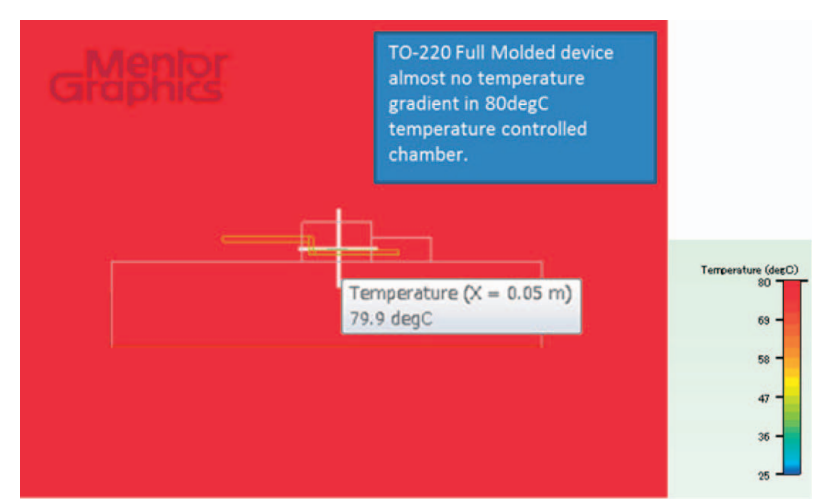

Fig. 16 Temperature gradient of TO-220 full molded package, in a temperature controlled chamber.

Interestingly, even the worst difference yield in this experiment with TO-220 full molded device, the error was less than $5 \%$ (3.6\%). It was smaller than we anticipated.

As the temperature gradient is shown in Fig. 15, this error is mainly due to the temperature gradient generated under single sided cold plate. Figure 16 shows that there is almost no temperature gradient when the device is in a temperature controlled chamber.

The common sense that the single sided cold plate result in worse TSP calibration results more than $10 \%$, might have due to the shorter $t_{T W}$ which doesn't meet the condition of Eq. (7). If the $t_{T W}$ is too short, even though the 
temperature controlled chamber or a chiller attached to the single sided cold plate has reached to the target temperature, the device under test can't catch up and cause a significant temperature differences. These significant errors could be avoided if the temperature of the cold plate, close to the device under test is monitored.

In this experiment, TSP difference decreases as $R_{t h-j c}$ decreases, as expected (except TO-264). Also, the bigger the package is, the lower the TSP difference became. The area of the device facing the cold plate is contributing to have less error.

\section{Conclusion}

In this paper, we discussed how the TSP calibration results differ due to the calibration environment. By using a single sided cold plate, the larger $R_{t h-j c}$ results in larger errors compared to the temperature controlled chamber. Since recent power devices have less than $0.5(\mathrm{~K} / \mathrm{W})$, it is acceptable to use a single sided cold plate. By monitoring the temperature of the cold plate close to the device would result in a better calibration results.

The method to obtain $\tau$ from junction of the device to the chamber/single sided cold plate was introduced in order to acquire appropriate sufficient $t_{T W}$ for the TSP calibration.

The TO-264 package showed relatively larger errors compared to TO-220, which has larger $R_{t h-j c}$, and SOT-227, which has similar $R_{t h-j c}$. This is left undiscussed in this paper.

\section{References}

[1] M. Rencz, V. Székely, A. Poppe, G. Farkas, and B. Courtois, "New methods and supporting tools for the thermal transient testing of packages," APACK 2001, Singapore, pp. 407-411, 2001.

[2] "Transient dual interface test method for the measurement of the thermal resistance junction to case of semiconductor devices with heat flow through a single path,” JEDEC Standard, JESD51-14, November, 2010, www.jedec.org.

[3] Y. Luo, Y. Kajita, T. Hatakeyama, S. Nakagawa, and M. Ishizuka, "Thermal transient test based thermal structure function analysis of IGBT package,” ICEP2014, Toyama, Japan, pp. 596-599, April 2014.

[4] Z. Sarkany, A. Vass-Varnai, and M. Rencz, "Investigation of die-attach degradation using power cycling tests,” EPTC2013, Singapore, pp. 780-784, December 2013.
[5] Z. Sarkany, A. Vass-Varnai, S. Laky, and M. Rencz, "Thermal transient analysis of semiconductor device degradation in power cycling reliability tests with variable control strategies,” SEMI-THERM2014, San Jose, CA, USA, pp. 236-241, 2014.

[6] Integerated circuits thermal measurement method Electrical Test Method (single semiconductor device), JEDEC Standard, JESD51-1, December, 1995, www.jedec.org.

[7] N. Marcano, A. Singh, and F. Perez, "Voltage-temperature characteristics of $\mathrm{W} / \mathrm{n}-\mathrm{GaAs}$ schottky diodes activated by the constant forward current: application as temperature sensors,” Devices, Circuits and Systems. ICCDCS 98, Isla de Margarita, Venezuela, pp. 88-91, 1998.

[8] N. Li and P. Jin, "Voltage-temperature coefficient analysis and testing of high power light-emitting diodes," Solid State Lighting (ChinaSSL), Beijing, China, pp. 137-141, November 2013.

[9] Y. Xi and E. F. Schubert, "Junction-temperature measurements in GaN UV light-emitting diodes using the diode forward voltage," High Performance Device, pp. 84-89, August 2004.

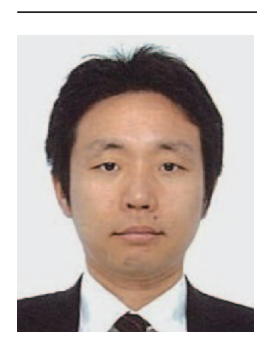

Tomoaki Hara was born in Chiba, Japan. He received B.S. degree and M.E. degree from Tokyo Institute of Technology in 2001 and 2003 respectively. He worked with Keyence Corporation for 10 years (spent one year working with Keyence Corporation of America). Now he works with Mentor Graphics Japan Co., Ltd. as Senior Application Engineer of Mechanical Analysis channel.

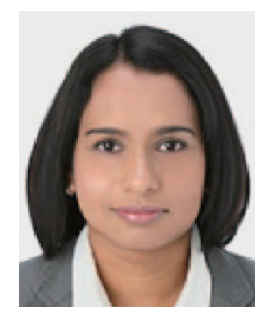

Wasanthamala Badalawa was born in Sri Lanka. She received M.S. and Ph.D. degrees in Electrical Engineering and Information Systems from the University of Tokyo, Japan, in 2007 and 2012 respectively. In 2012 she joined the Advantest cooperation and developed CMOS Timing Generators for 2 years 6 months. She is currently working in the Mechanical analysis division of Mentor Graphics Japan Co., Ltd.

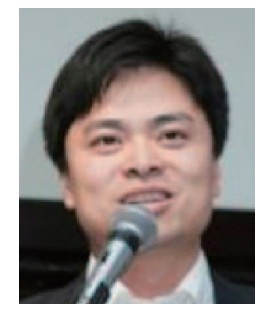

Yafei Luo was born in 1980, China. Graduated from Peking University with B.S. degree in 2003 and obtained M.E. degree in Florida International University in 2005 . He has 5 years' experience of ASIC design/verification and now his work is focusing on thermal measurement and analysis as application engineer manager in Mentor Graphics Japan Co., Ltd. 